\title{
Evidence Synthesis for Medical Decision Making and the Appropriate Use of Quality Scores
}

\author{
Suhail A. R. Doi, MBBS, FRCP, MClinEpid, PhD
}

\begin{abstract}
Meta-analyses today continue to be run using conventional random-effects models that ignore tangible information from studies such as the quality of the studies involved, despite the expectation that results of better quality studies reflect more valid results. Previous research has suggested that quality scores derived from such quality appraisals are unlikely to be useful in meta-analysis, because they would produce biased estimates of effects that are unlikely to be offset by a variance reduction within the studied models. However, previous discussions took place in the context of such scores viewed in terms of their ability to maximize their association with both the magnitude and direction of bias. In this review, another look is taken at this concept, this time asserting that probabilistic bias quantification is not possible or even required of quality scores when used in meta-analysis for redistribution of weights. The use of such a model is contrasted with the conventional random effects model of metaanalysis to demonstrate why the latter is inadequate in the face of a properly specified quality score weighting method.
\end{abstract}

Keywords: Bias; Medical decision making; Meta-analysis; Quality scores

\begin{abstract}
A common problem with meta-analyses is that they combine results from studies that may be quite heterogeneous (in terms of the effect estimate) and efforts have been made to account for this heterogeneity in meta-analytic summaries. One common method of dealing with this has been through study "quality appraisals" that usually involve quantifying the number of study deficiencies according to a pre-specified number of components (items) that are reported in or can be inferred from the published paper. The premise is that a poor quality study may lead to systematic bias in the estimate of a treatment's effectiveness, if it avoids protecting the study from threats to internal validity. ${ }^{1}$ In addition, the number of non-faulty quality traits are presumed to predict the extent to which aspects of a study's design and conduct protect against biases and inferential errors. ${ }^{2}$ Although such quality assessment has sometimes been extended beyond the appraisal of internal validity, in this discussion reference will
\end{abstract}

only be made to the latter - how well the study was designed and executed to prevent systematic errors or bias. ${ }^{3}$ If the quality items or traits are assigned a number of points based on the a priori judgment of clinical investigators, they can then be summed into a univariate "quality score" that has previously been given the interpretation of capturing the essential features of a "multidimensional quality space"3,4 and has been used as a weighting factor in averaging across studies. ${ }^{5-7}$ or as a covariate for stratifying study results. ${ }^{8,9}$

Greenland has raised scientific objections to the creation of this sort of univariate quality score from quality items., ${ }^{410,11}$ This seems to be supported by empirical findings of deficiencies when stratifying by such scores ${ }^{8}$ or when associating them with variations in treatment effects, ${ }^{12,13}$ although there are also reports of beneficial inferences through such scores. ${ }^{3,14}$ Greenland suggests that the rationale

Received: July 22, 2013

Revised: August 27, 2013

Accepted: September 5, 2013

doi: $|0.3| 2|/ \mathrm{cmr} .20| 3.1 \mid 88$ 
behind their use as weights in meta-analysis lacks rigorous justifications and may even be in error and proposed instead direct modeling of component item quality. ${ }^{4}$ In agreement with Greenland, there has been the emergence of component item quality modeling schemes, ${ }^{15,16}$ but this paper will attempt to show that, in general, correct quality-score weighting methods can produce less variable effect estimates, as has been suggested by some authors who continue to use and even promote such scores..$^{6,17,18}$ This justification stands, even when the component quality items do not capture all traits contributing to bias in study-specific estimates. Furthermore, an attempt will be made to demonstrate if the possible decrease in variance with such quality weighting can be a worthwhile trade-off against bias and how it performs against the random effect methods. It will be argued that qualityscore weighting methods take precedence over direct modeling of quality dimensions, ${ }^{15}$ in part because the impact of a quality dimension on the direction or magnitude of an effect estimate are unknowns. It will also be demonstrated that for quality weights to work well only requires the quality score to be constructed to maximize its correlation with study deficiencies that have an impact on study effects, rather than any requirement for the quality score to be a specific function of quantitatively measured bias.

\section{Bias, Quality and Meta-Analysis Models}

The following evaluation starts off, as suggested by Greenland and O'Rourke, ${ }^{4}$ with a response-surface model for metaanalysis where the expected study-specific effect estimate is a function of the true effect in the study, plus bias terms that are influenced by quality items. Exactly the same terminology and notation as used by Greenland and O'Rourke will be used to make it clear to readers where the divergence is; so let $i=$ $1, \ldots, I$ index studies, and let $X$ be a row vector of $J$ quality items. For example, $X$ could contain indicators of quality of the design or conduct of a study, such as use of blinding or allocation concealment in meta-analyses of randomized trials, or measures taken to avoid confounding in meta-analyses of observational studies. Similarly, $\delta_{i}=$ expected study-specific effect estimate; $\theta=$ the parameter that the meta-analysis aims to estimate (which is the true but unknown effect of study treatment), and $\chi_{i}=$ value of $X$ for study $i$. The study-specific estimate $\delta_{i}$, is the effect size actually reported in study $i$ (such as a standardized mean difference). If $\chi_{o}$ is the value of $X$ that is a priori taken to represent the "best quality" with respect to the measured quality items, then the response-surface approach $^{4}$ has been used to model $\delta_{i}$ as a regression function of $\theta$ and $\chi_{i}$, and a simple fixed effects additive model would be:

$$
\delta_{i}=\theta+b_{i}
$$

where $b_{i}$ is the bias resulting in deviation of $\delta_{i}$ from the true value $\theta$, and which has previously been interpreted as being associated with $\chi_{i}$ deviating from the ideal $\chi_{o}$, with this ideal supposedly implying lack of bias. In reality, this model is constrained by the fact that quality scoring does not specify the value of $b_{i}$ accurately, in the sense that even when a study has an ideal value $\chi_{o}$, there would still be a bias component of

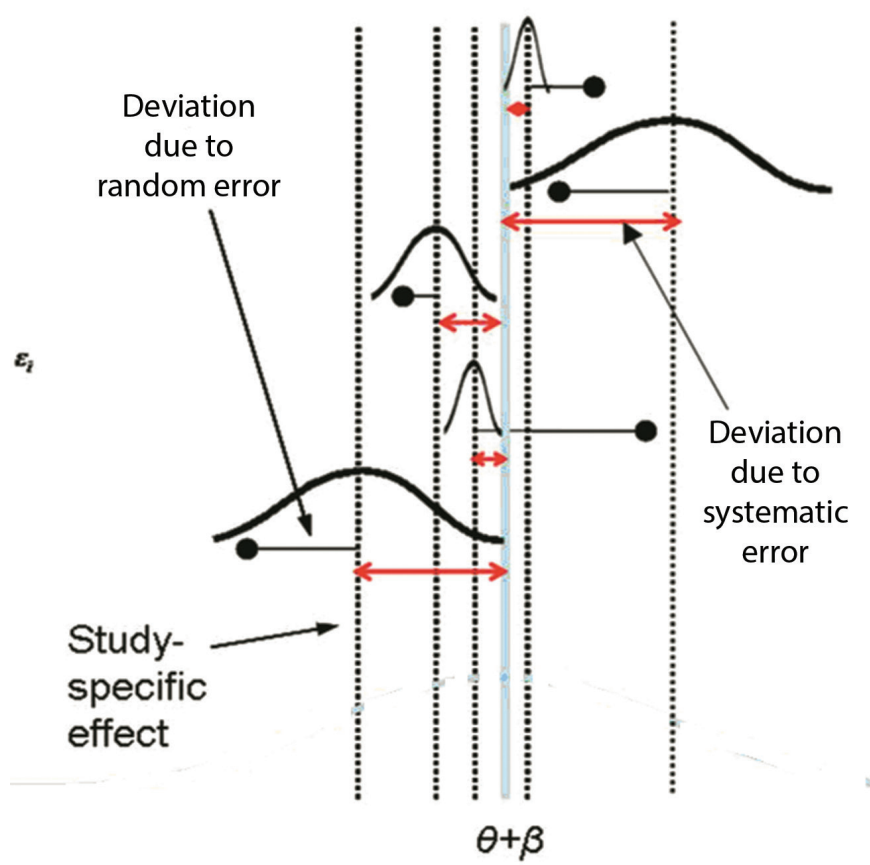

Figure 1. Conceptualization of model 2.

$\delta_{i}$ that is due either to other unmeasured quality items or that is poorly measured by the quality assessment scale or both (ie, $X$ cannot be expected to capture all bias information). In this situation, and because there are only a few $(I)$ observations $\hat{\delta}_{i}, \ldots, \hat{\delta}_{i}$, in most meta-analyses, these data constraints need to be overcome by specifying a more appropriate model. Current methods do so by employing models in which neither $b_{i}$ nor its component effects are present and, therefore, in order to capture more uncertainty than fixed effect models, resort to constraining the underlying effect of interest to be a random effect drawn from a fictional distribution with unknown mean and variance. These models have been criticized for the fictional assumption that there is a random sample of studies in a meta-analysis ${ }^{19}$ or ignoring study traits such as differences in the way in which treatment protocols were designed or conducted. ${ }^{6,20}$ So, rather than add a fictional residual random treatment effect to the model, this paper diverges from other authors ${ }^{4,7}$ by assuming that while the $\theta$ can be treated as fixed, the effects of bias on the uncertainty of estimates can be included by the creation of a synthetic standard error that includes a component of variance for between study bias, ${ }^{21,22}$ and thus, model 1 becomes

$$
\delta_{i}=\theta+b_{i}=\theta+\beta+\varepsilon_{i}
$$

Under model $2, E\left(\delta_{i}\right)=\theta+\beta, E\left(\varepsilon_{i}\right)=0, \operatorname{var}\left(\varepsilon_{s i}\right)=\phi_{i}^{2}$ with $s$ indexing study subjects, $b_{i} \sim N\left(\beta, \phi^{2}\right)$ and $\beta$ (the superpopulation bias across studies), though expected to be zero, may not cancel across studies if systematic biases were predominantly in one direction. It is unlikely that under model 2, the study specific bias effects, $\varepsilon_{i}$ and the sampling error residuals $\hat{\delta}_{i}-\delta_{i}$ are correlated, but even so, $\chi_{i}$ cannot be used to estimate $\theta$ from the bias-adjusted estimates $\hat{\delta}-b_{i}$, since measurement of $b_{i}$ cannot be achieved through interpretation of $\chi_{i}$. Some researchers have suggested that quality reflects the within study variability that is unrelated to 
internal biases, ${ }^{16}$ ie, $v_{i} /\left(v_{i}+\phi_{i}{ }^{2}\right)$, where $v_{i}=\operatorname{var}\left(\hat{\delta}_{i} \mid \delta_{i}\right)$, but this seems to contradict the type of information available from quality appraisal. Rather, from model $2, \chi_{i}$ can be expected to reflect the proportion of the total bias variability that is unrelated to internal study biases, ie, $\phi^{2} /\left(\phi^{2}+\phi_{i}^{2}\right)$. There is then the expectation that the model would improve if $\chi_{i}$ can be used to redistribute weights, such that it reduces the variance of the weighted estimator, or in the case of non-zero $\beta$, reduces it far enough to counter the increase in bias. This model is depicted in figure 1, and it is of interest to note that bias variances need not be known for this model use of information contained in $X$.

\section{Methodology of Quality Weights}

As described by Greenland, ${ }^{4}$ to create a univariate score, the vector of quality items $X$ can be replaced with a unidimensional scoring rule $\mathrm{s}(X)$, which is usually a weighted sum $X q=\sum_{j} q_{j}$ $X_{j}$ of the $J$ quality items in $X$, where q is a column vector of "quality weights" specified a priori and commonly taken to be equal in the absence of specific weighting data. This score can then be re-scaled between 0 and 1 and is interpreted as an intraclass correlation. Since we do not know the value of the bias variances, we divide $s_{i}$ by the maximum $s_{i}$ in the list of studies resulting in the same minimum and maximum quality scores $Q_{i}$ for any a priori specified function. The quality scores, $Q_{i}$, are then used for quality weighting by averaging the study-specific estimates, $\hat{\delta}_{i}$, after multiplying the usual inverse-variance weight by $Q_{i}$. For example, even though the unconditional variance of $\hat{\delta}_{i}^{i}$ is $v_{i}+\phi_{i}^{2}$, the usual inverse variance weight for $\delta_{i}$ would be $\hat{\mathrm{w}}_{i}=1 / v_{i}$ (since $\phi_{i}^{2}$ is unknown), and the "quality-adjusted" weight is $Q_{i} \mathrm{w}_{i} \cdot{ }^{6}$ If the quality score is conceptualized as maintaining a monotonic relationship with the ratio of between to total bias variance, it can then be used to redistribute study weights away from studies of lesser quality and thus greater internal "noise." The latter weights can be expected to decrease variance of the weighted estimator, because $s_{i} \equiv \phi^{2} /\left(\phi^{2}+\phi_{i}^{2}\right)^{23}$ and, thus, the proportional contribution of $\phi_{i}^{2}$ to total bias (in the context of the studies within the meta-analysis of interest) declines in a monotonic fashion as $s_{i}$ increases from zero to 1 .

This sort of weighting does not require $X$ to contain all biasrelated covariates, and the quality score need not be a function of quantitatively measured bias. Also, $Q_{i}=1$ does not imply the absence of study deficiencies, but rather the relative strength (rank) of the study given the data at hand. To see how this works, the same example given by Greenland ${ }^{4}$ can be taken. Let $u_{i}$ be any study-specific weight and let a subscript $u$ denote averaging over studies using the $u_{i}$ as weights then for example:

Under model 2,

$$
\delta_{u}=\sum u_{i} \delta_{i} / \sum u_{i}
$$

$$
\delta_{u}=\theta+b_{u}=\theta+\beta+\varepsilon_{u}
$$

Thus, $\delta_{u}$ equals the true effect $\theta$ plus external bias across studies (if any) plus the u-average internal bias $\varepsilon_{u}$. Therefore, any unbiased estimator of $\delta_{u}$ will certainly be biased for $\theta$ in those situations where the super-population bias across studies does not cancel and thus $\beta \neq 0$. The expectation, however, is that even under the latter constraint, such bias will be overshadowed by the expected decrease in overall variance of this weighted estimator such that an optimal biasvariance trade-off can be reached. The coverage properties of the estimators CI has further been augmented by creating a system that redistributes weights from lower scoring studies to other studies proportional to their values on $Q_{i}{ }^{17}$ All that is required of the quality scores in this model is that they are a monotone function of the safeguards in a study and so rank the studies with respect to safeguards (rather than be any fixed function of bias). ${ }^{6}$ It is important that such identified safeguards have a clear link to systematic error, and so cannot be ethical concerns or attributes linked solely to precision. The use of quality weighting in this way means that the two assumptions outlined by Greenland ${ }^{4}$ are acknowledged: (a) that there is useful information in studies of less-thanmaximal quality, and that some degree of bias is tolerable if it comes with a large variance reduction, and (b) that errors due to bias are not considered more costly than errors due to chance, as would be implicitly assumed had we limited our attention to unbiased estimators.

\section{Issues with Quality Scoring}

An important issue where care must be taken is to ensure that the quality scoring rule contains items relevant to systematic bias (eg, excludes items related to whether power calculations were reported or ethics was properly sought), as such items will disproportionately reduce the impact of the resulting scores, even if conceptualized as deficiency scores given that these deficiencies must still link to systematic error. Furthermore, inclusion of such items can distort deficiency rankings of the studies as well.

Another issue is that given that the scoring rule $\mathrm{s}(X)$ is usually a weighted sum of the $J$ quality items in $X$, where $q$ is a column vector of "quality weights" there should be special attention given to determining the proper weight $q$ that an item should have. In the absence of such weighting data, $q$ is currently taken to be an equal weight across items. There is thus an urgent need to generate this information from metaepidemiological studies.

Greenland has alluded to "score validations" as being typically circular, ${ }^{4}$ in that the criteria for validation hinge on score agreement with opinions about which studies are "high quality," not on actual measurements of bias. This argument is no longer circular if score measurements are viewed in the context of study deficiencies, since actual measurement of bias is no longer being sought through quality scores. Greenland also suggests that perhaps the worst problem with common quality scores is that they fail to account for the direction of bias induced by quality deficiencies. ${ }^{10}$ While this failing can nullify the value of a quality score in regression analyses based on the bias modeling outlined by Greenland, ${ }^{4}$ not so in quality weighted meta-analyses (as defined here), as neither magnitude nor direction of bias are being assessed. In 
Table 1. Summary of simulation parameters. *

\begin{tabular}{|c|c|c|c|}
\hline Simulation & Model parameters & Study parameters & $\begin{array}{l}\text { Values allocated through } \\
\text { simulation }\end{array}$ \\
\hline 1 & $\begin{array}{l}\theta=0.5 \\
\beta=0 \\
\phi^{2}=0.15\end{array}$ & $\begin{array}{l}s_{i} \text { fixed at } 0.1,0.2,0.5,0.8 \\
\text { and } 0.9 \text { for studies } 1 \text { to } 5 \\
\text { respectively } \\
\delta_{i}=0.5+\beta+\varepsilon_{i} \\
\phi_{i}^{2} \approx\left(\phi^{2}-\phi^{2} s_{i}\right) / s_{i} \\
v_{i}=\frac{4}{N}+\frac{\hat{\delta}_{i}^{2}}{2 N}\end{array}$ & $\begin{array}{l}N \text { was randomly allocated } \\
\text { between } 50 \text { and } 1000 \text { in steps } \\
\text { of } 50 \text { with } N / 2 \text { in each group } \\
\text { (intervention/control) } \\
\delta_{i} \sim N\left(0.5, \phi_{i}^{2}\right) \\
\delta_{i} \sim N\left(\delta_{i}, \frac{4}{N}+{\frac{0.5^{2}}{2 N}}^{2}\right)\end{array}$ \\
\hline 2 & $\begin{array}{l}\text { Same as } \\
\text { simulation (1) }\end{array}$ & $\begin{array}{l}Q_{\mathrm{i}} \text { randomly allocated } \\
\text { between } 0.1 \text { and } 0.9 . \\
\text { Rest same as simulation (1) }\end{array}$ & Same as simulation (1) \\
\hline 3 & $\beta=0.1$ & Same as simulation (1) & $\delta_{i} \sim N\left(0.6, \phi_{i}^{2}\right)$ \\
\hline & $\begin{array}{l}\text { Rest same as } \\
\text { simulation (1) }\end{array}$ & & Rest same as simulation (1) \\
\hline
\end{tabular}

${ }^{*}$ performance measures used are defined in Burton et al ${ }^{28}$

this paper, the pragmatic view is being taken that a study with two or more identified deficiencies can be afflicted by more bias variance than one with none regardless of the magnitude or direction of the resulting bias. Thus, bias cancellation phenomena are not relevant when quality scores are conceptualized of as deficiency scores, and there is also no need to reconstruct a quality score as a bias score with signs attached to the contributing items as illustrated by Greenland ${ }^{4}$ or by Thomson et al. ${ }^{15}$ Indeed, the amount of bias expected $a$ priori and their directions are unknowns that cannot and should not be imputed when constructing the quality scores. Hence, reconstructing the scores to maximize their correlation with bias ${ }^{4,15}$ does not appear to be necessary or essential for the intelligent use of quality scores in properly specified quality effects models such as that described in this paper or reported by others. ${ }^{17,18}$

Finally, how the proposed model for quality scores could be used to further clinical decision support through research synthesis is, in general, a bigger question and an area of ongoing controversy. It would be envisaged that in the future, special interest groups could take the lead in quality scoring all the pertinent literature in their field based on their own consensus criteria. Currently, this is being done by the PEDro database owners, and recently they were criticized for this, ${ }^{24}$ but we were quick to respond to this criticism in their support. ${ }^{25}$ The availability and acceptance of models described in this paper should help pave the way for more widespread support for such endeavors in the near future.

A Comparison of Quality and Random Effect Models A simulation can be set up under model 2 by assigning a value $\theta=0.5$, representing a standardized mean difference effect size. Five hypothetical studies can be generated by incorporating $\mathrm{N}$ randomly allocated subjects (constrained between 50 and 1000 in steps of 50 by generating a random integer between 1 and 20 and multiplying by 50) in each study and assuming N/2 are in the treatment and control groups respectively for simplicity. Bias, $b_{i}$, is generated and added to each study by arbitrarily assigning $\beta=0$ (making the assumption that external biases across studies cancel, thus resulting in an unbiased estimator) and fixing $\phi^{2}$ at an arbitrary 0.15 , which is about 10 -fold higher than the average $v_{i}$ based on the true effect (0.5) and study size (N) (to make the studies heterogeneous). Since the external bias variance for the meta-analysis has been fixed, and if the probability of credibility or quality $\left(s_{i}\right)$ input for each study is also fixed, this can then be used to simulate the individual study bias variances since $\phi_{i}^{2} \approx\left(\phi^{2}-\phi^{2} s_{i}\right) / s_{i}$. The values of were fixed at $0.1,0.2,0.5,0.8$ and 0.9 for studies 1 to 5 respectively. Even though latter quality input is fixed, because the actual $\phi^{2}$ value for each set of studies will differ from the starting value, the actual quality also differs from these values (though rank by quality remains the same). This adds the required "subjectivity" in quality assessment to the analysis.

Thus, with the true underlying parameter of interest fixed at 0.5 , then for each study $\mathrm{i}=1, \ldots, 5, \delta_{i}=0.5+\beta+\varepsilon_{i}$ can be generated by sampling $\varepsilon_{i}$ from a normal distribution with mean $0.5(\beta=0)$ and variance $\phi_{i}^{2}$. The final study estimate $\hat{\delta}_{i}$, is then determined by adding sampling error to $\delta_{i}$ by generating this error from a normal distribution with mean zero and variance $v_{E S=0.5}=\frac{4}{N}+\frac{0.5^{2}}{2 N}$. A set of five studies are finally created with effect size $\delta_{i}$ and variance $v_{i}=\frac{4}{N}+\frac{\hat{\delta}_{i}^{2}}{2 N}$, and this set of studies is then the simulation set. To generate the study estimates for each iteration, a simulation software (Ersatz version 1.3; Epigear International Pty Ltd) was used. Essentially, each simulation underwent 50,000 iterations, and at each iteration first created five studies, then meta-analyzed them and finally computed the input for the performance measures and stored these. Then, the next iteration did the 

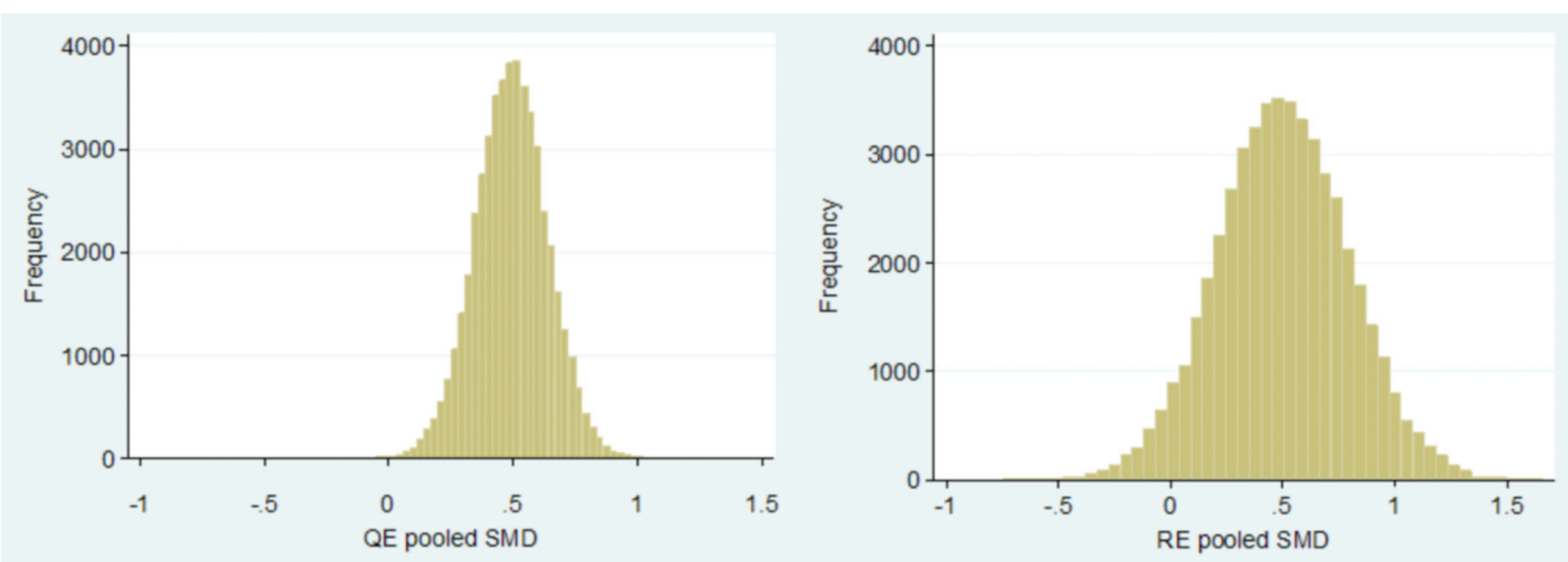

Figure 2. Comparison of the distribution of pooled estimates by the quality effects (left panel) and random effects (right panel) models when $\beta=0$ and quality is not-mis-specified (simulation 1).

same and so on until 50,000 iterations were completed. The meta-analysis estimates computed under each iteration for both the random and quality effects methods used another plug-in (MetaXL version 1.3, Epigear International Pty Ltd) that includes the analogous quality effects (QE) model of Doi and Thalib ${ }^{6,26}$ and the random effects (RE) model of Dersimonian and Laird. ${ }^{27}$ Performance measures were then computed as detailed by Burton et al. ${ }^{28}$ This process was then repeated, in run 2 with $\beta=0$ but randomly mis-specified quality input into the quality effects model and then, in run 3 for $\beta=0.1$ (and properly specified quality) to simulate $20 \%$ external bias across the latter studies. Model and study parameters are summarized in Table 1 across the three simulation runs.

Results were exactly as expected. With $\beta=0$ and properly specified quality, the RE model had a MSE (0.0862) that was four-fold higher than the MSE (0.0212) of the QE model (figure 2). However, there was not much bias that was similar with the QE (-1.1\%) compared to the RE model $(0.09 \%)$. Coverage probability and CI width were better at $97.6 \%$ and 0.81 with the QE model compared to $89.7 \%$ and 0.99 , respectively, with the RE model. In addition, $76 \%$ of the pooled estimates were closer to the parameter of interest with the $\mathrm{QE}$ as compared to the RE model.

When $Q_{i}$ was mis-specified at the point of meta-analysis only, (replaced by a random quality between 0.1 and 0.9 by generating a random integer between 1 and 9 and dividing by 10), the QE model results now became identical to that of the RE model (MSE 0.10 vs 0.09; figure 3, top right panel). The latter is because the QE model with random misspecification simply defaults to a RE model. When super-population bias was constrained to a non-zero value $(0.1)$, but with proper specification of quality (figure 3, lower panel), both models were equally biased (18.8\% [0.094] QE vs $19.6 \%$ [0.098] $\mathrm{RE})$, but the QE model had a much better bias-variance tradeoff (MSE 0.0299 vs 0.0959). Coverage of the CI and CI width were $95 \%$ and 0.81 with the QE model compared to $85.5 \%$ and 0.99 with the RE model. This is depicted in the lower panel of figure 3. While these simulation results presented here need to be reproduced in wide-spread real world applications, they fit well with the theoretical model, and thus there is every expectation of similar results with real world applications.

\section{Conclusion}

The random effects model, with the assumption that the true effect $\theta$ varies in a simple random manner across studies, has no credibility ${ }^{4,29}$ and, therefore, the quality weighted model analogous to the theory outlined above addresses this by allowing bias to vary and redistributing weights through quality based on the expected bias variance differences across studies. In this case, however, the quality score is no longer conceptualized as a bias score, but rather a deficiency score, and this is necessary because the impact of quality dimensions on the direction and magnitude of bias are unknowns. However, Greenland visualized quality information as a means to specifying bias magnitude and direction quantitatively, and was thus forced to conclude that common quality score methods are both biased as well as require special cases of mixed effect meta-regression models. ${ }^{4}$ Currently, there exists one model that implements the biasvariance concept through quality scores, and it is demonstrated here via simulation that under all constraints, it would certainly represent a model with a better bias-variance tradeoff than the random effects model. It is also demonstrated here that the random effects model is simply a quality effects model, where quality is randomly assigned to studies (a misspecified quality effects model) and nothing more. This then obviates the common argument regarding the subjectivity of quality scoring, since in the worst case scenario (a completely random assignation of the quality score to studies), the performance of both models converge. The latter precludes criticism regarding the subjectivity of the quality scores, because so long as they contain some information value, the quality effects estimator will always outperform the random effects estimator. Improvements to the quality effects model 
can be expected to occur as quality assessment tools become more objective and validated over time, and more information accrues regarding the appropriate weighting of quality items within a univariate score. It, therefore, seems that the time is right to herald the discontinuation of such "random effect" models in the face of better alternatives and available software. It is hoped that this would herald a new era of more reliable research synthesis results through meta-analysis.

\section{References}

1. Detsky AS, Naylor CD, O'Rourke K, McGeer AJ, L'Abbe KA. Incorporating variations in the quality of individual randomized trials into meta-analysis. J Clin Epidemiol 1992;45:255-265.

2. Lohr KN. Rating the strength of scientific evidence: relevance for quality improvement programs. Int J Qual Health Care 2004;16:9-18.

3. Hartling L, Ospina M, Liang Y, Dryden DM, Hooton N, Krebs Seida J, Klassen. Risk of bias versus quality assessment of randomised controlled trials: cross sectional study. BMJ 2009;339:b4012

4. Greenland S, O'Rourke K. On the bias produced by quality scores in meta-analysis, and a hierarchical view of proposed solutions. Biostatistics 2001;2:463-471.
5. Moher D, Pham B, Jones A, Cook DJ, Jadad AR, Moher M, Tugwell P, Klassen TP. Does quality of reports of randomised trials affect estimates of intervention efficacy reported in meta-analyses? Lancet 1998;352:609-613.

6. Doi SA, Thalib L. A quality-effects model for meta-analysis. Epidemiology 2008;19:94-100.

7. Berard A, Bravo G. Combining studies using effect sizes and quality scores: application to bone loss in postmenopausal women. J Clin Epidemiol 1998;51:801-807.

8. Juni P, Witschi A, Bloch R, Egger M. The hazards of scoring the quality of clinical trials for meta-analysis. JAMA 1999;282:1054-1060.

9. Herbison P, Hay-Smith J, Gillespie WJ. Adjustment of metaanalyses on the basis of quality scores should be abandoned. J Clin Epidemiol 2006;59:1249-1256.

10. Greenland S. Quality scores are useless and potentially misleading: Reply to: re: a critical look at some popular analytic methods. Am J Epidemiol 1994;140:300-301.

11. Greenland S. Invited commentary: a critical look at some popular meta-analytic methods. Am J Epidemiol 1994;140:290-296.

12. Emerson JD, Burdick E, Hoaglin DC, Mosteller F, Chalmers TC. An empirical study of the possible relation of treatment differences to quality scores in controlled randomized clinical trials. Control Clin Trials 1990;11:339-352.
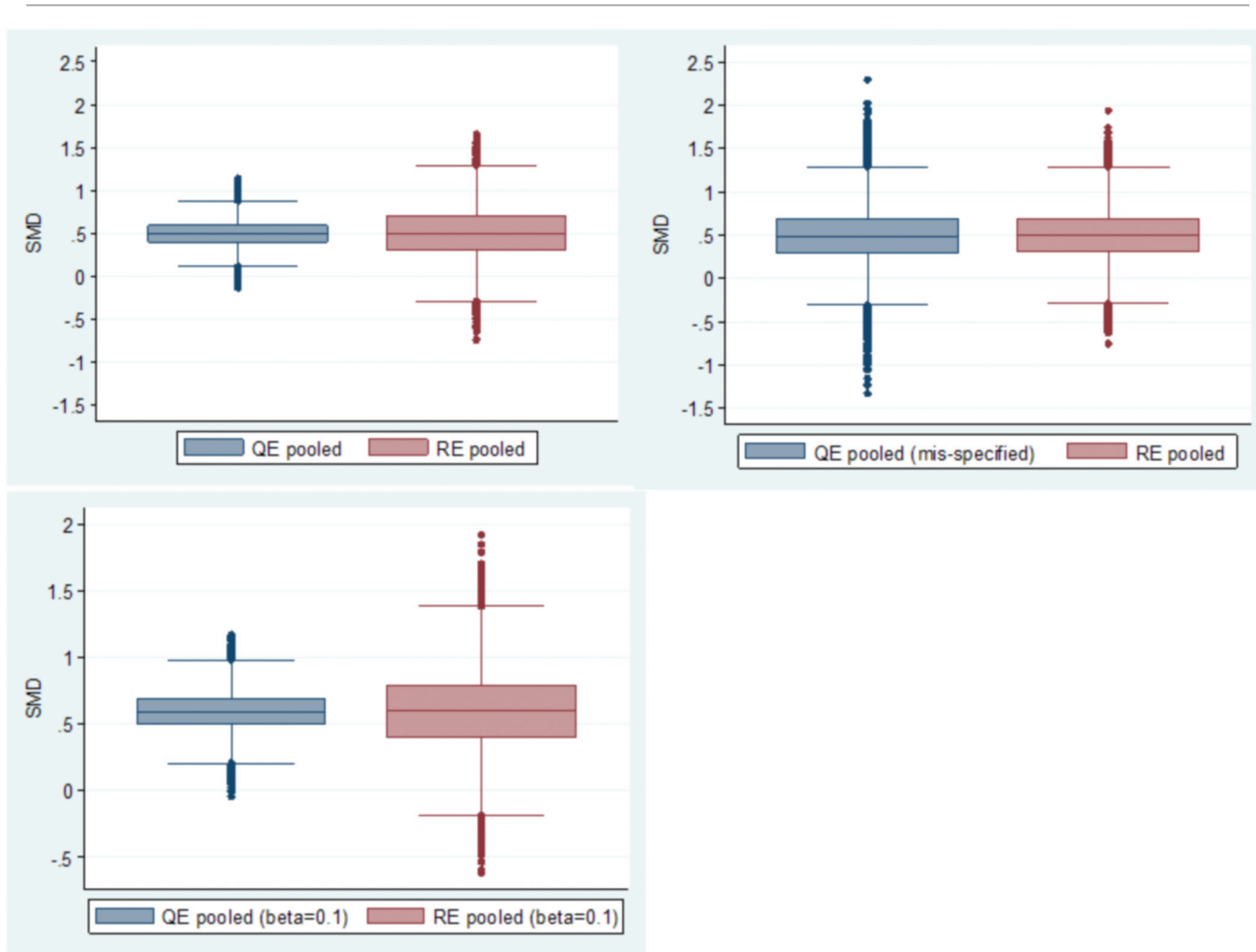

Figure 3. Comparison of the distribution of pooled estimates by the quality effects model when $\beta=0$ (top left panel; simulation 1) and mis-specified quality effects model when $\beta=0$ (top right panel; simulation 2) in comparison to the RE model. The bottom panel (simulation 3 ) represents the constraint that $\beta=0.1$. Each panel represents a separate simulation run with 50,000 iterations involving 250,000 individual studies. 
13. Balk EM, Bonis PA, Moskowitz H, Schmid CH, Ioannidis JP, Wang C, Lau J. Correlation of quality measures with estimates of treatment effect in meta-analyses of randomized controlled trials. JAMA 2002;287:2973-2982.

14. Kjaergard LL, Villumsen J, Gluud C. Reported methodologic quality and discrepancies between large and small randomized trials in meta-analyses. Ann Intern Med 2001;135:982-989.

15. Thompson S, Ekelund U, Jebb S, Lindroos AK, Mander A, Sharp S, Turner R, Wilks D. A proposed method of bias adjustment for meta-analyses of published observational studies. Int J Epidemiol 2011;40:765-777.

16. Turner RM, Spiegelhalter DJ, Smith GC, Thompson SG. Bias modelling in evidence synthesis. J R Stat Soc Ser A Stat Soc 2009;172:21-47.

17. Doi SA, Barendregt JJ, Onitilo AA. Methods for the bias adjustment of meta-analyses of published observational studies. J Eval Clin Pract 2013;19:653-657.

18. Doi SA, Barendregt JJ, Mozurkewich EL. Meta-analysis Of heterogenous clinical trials: an empirical example. Contemp Clin Trials 2011;32:288-298.

19. Overton RC. A comparison of fixed-effects and mixed (random-effects) models for meta-analysis tests of moderator variable effects. Psychological Methods 1998;3:354-379.

20. Peto R. Why do we need systematic overviews of randomized trials? Stat Med 1987;6:233-244.

21. Draper D. Assessment and propagation of model uncertainty. J R Statist Soc B 1995;57:45-97.

22. Draper D, Gaver DP, Goel PK, Greenhouse JB, Hedges LV, Morris CN, Tucker JR, Waternaux C. Combining information: statistical issues and opportunities for research. Washington, DC: National Research Council; 1993.

23. Spiegelhalter DJ, Best NG. Bayesian approaches to multiple sources of evidence and uncertainty in complex costeffectiveness modelling. Stat Med 2003;22:3687-3709.

24. da Costa BR, Hilfiker R, Egger M. PEDro's bias: summary quality scores should not be used in meta-analysis. J Clin Epidemiol 2013;66:75-77.

25. Doi SA, Barendregt JJ. Not PEDro's bias: summary quality scores can be used in meta-analysis. J Clin Epidemiol 2013;66:940-941.

26. Doi SA, Thalib L. An alternative quality adjustor for the quality effects model for meta-analysis. Epidemiology 2009;20:314

27. DerSimonian R, Laird N. Meta-analysis in clinical trials. Control Clin Trials 1986;7:177-188.

28. Burton A, Altman DG, Royston P, Holder RL. The design of simulation studies in medical statistics. Stat Med 2006;25:4279-4792.

29. Poole C, Greenland S. Random-effects meta-analyses are not always conservative. Am J Epidemiol 1999;150:469-475.

\section{Author Affiliation}

Suhail A. R. Doi MBBS; FRCP; MClinEpid, PhD; Associate

Professor of Clinical Epidemiology, School of Population Health,

University of Queensland, Brisbane, Australia 